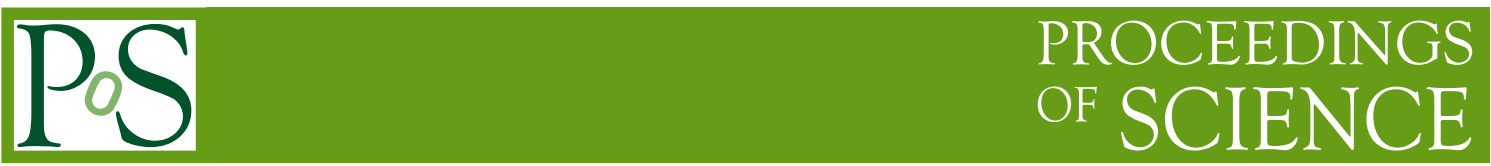

\title{
Uncovering the radio emission of low-mass systems
}

\author{
Juan B. Climent ${ }^{a, *}$ and J. C. Guirado, M. A. Pérez-Torres, M. R. Zapatero-Osorio, O. \\ Zakhozhay \\ ${ }^{a}$ Departament d'Astronomia i Astrofísica, Universitat de València \\ C. Dr. Moliner 50, 46100 Burjassot, València, Spain
}

E-mail: j.bautista.climent@uv.es

Radio observations allow us to probe the nature of low mass objects, including ultracool dwarfs. Additionally, very long baseline interferometric observations provide the highest angular resolution in astronomy to date, which opens a window to measure dynamical masses of these objects in order to benchmark evolutionary models. We present here a multi-epoch, multi-frequency study of the substellar triple system VHS 1256-1257. We have found radio emission at $6 \mathrm{GHz}$ and 33 $\mathrm{GHz}$ coincident with the expected position of the central binary of VHS 1256-1257. No emission is detected at higher frequencies $(230 \mathrm{GHz}$ and $345 \mathrm{GHz}$ ) nor at $5 \mathrm{GHz}$ with VLBI arrays. The emission appears to be stable over almost 3 years at $6 \mathrm{GHz}$. These results can be well explained by non-thermal gyrosynchrotron emission originating at radiation belts present in both components and viewed equatorially. We also share preliminary results from our VLA observations of 5 low-mass binary systems and EVN observations of 4 ultracool dwarfs (including a binary).

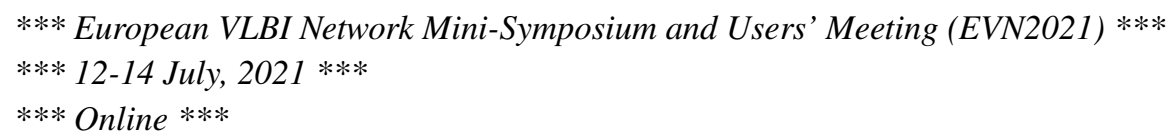

${ }^{*}$ Speaker 


\section{Introduction}

Radio observations of stellar or sub-stellar objects with masses $<0.7 \mathrm{M}_{\odot}$ play a crucial role in our understanding of their nature and emission mechanisms. At the very low mass regime, ultracool dwarfs (objects with spectral type later than M7; UCDs) cover the boundary between stars and exoplanets. The discovery of the first radio emission from an UCD proved the existence of powerful magnetic fields $(\sim \mathrm{kG})$ on these objects (Berger et al. 2001) which persist even in the coolest $\mathrm{T}$ dwarfs (Kao et al. 2018). Follow-up observations of UCDs at GHz frequencies have shown that $\sim 10 \%$ exhibit radio emission (Route \& Wolszczan 2016) that can be classified as quiescent (stable in time with low to moderate degree of circular polarization) or pulses (highly polarized, rapidly varying coherent bursts of radio emission). The former are usually attributed to gyrosynchrotron radiation (Berger 2002, Guirado et al. 2018) whereas the latter to the electron cyclotron maser instability (Hallinan et al. 2007, 2008; Kao et al. 2016; ECMI) linked to auroral emission.

From such observations, we are able to directly measure the strength and topology of the UCDs' magnetic fields which is crucial as the widely-used Zeeman spectroscopy technique requires very high signal-to-noise ratios and a low/moderate rotation velocities (Reiners \& Basri 2010), conditions that are hardly ever met in UCDs. Up to date, the majority of radio detections of UCDs have ocurred at $\mathrm{GHz}$ frequencies, with a few but notable exceptions like the first brown dwarf discovered at radio wavelengths (Vedantham et al. 2020).

Beyond the science that radio observations allow us to probe presently, the knowledge gathered from such observations may open a suitable route for the detection of exoplanetary radio emission, as well as emission resulting from the planetary interaction with the stellar wind (Turnpenney et al. 2018, 2020).

Of those UCDs emitters at radio frequencies, only three have been reported in the literature to present compact emission detectable with Very Long Baseline Interferometry (VLBI) arrays: TVLM 513-46546, for which a direct limit on the brightness temperature was established (Forbrich \& Berger 2009) and where, recently, the first exoplanet from radio astrometry has been discovered (Curiel et al. 2020); LSPM J1314+1320AB, where radio emission was found to be originating in only one of the components of the binary (Forbrich et al. 2016); and 2MASS J0746+2000AB, where both components of the binary system emit at radio emission and the most precise mass estimates of any UCDs to date could be obtained (Zhang et al. 2020). The latest spectral type with reported compact radio emission is an L3.5 object (Zhang et al. 2020).

Despite the noteworthy results, in-depth studies of UCD radio emission are still relatively scarce due, in part, to their difficult detection. New observations are needed to distinguish among the different proposed mechanisms for the origin of the strong magnetic fields and radio emission detected in these objects. As such, the system VHS J125601.92-125723.9 (hereafter VHS 1256-1257; Gauza et al. 2015) represents an excellent opportunity since its radio emission has been previously confirmed (Guirado et al. 2018) and new observations can provide further constraints on its origin. This nearby system $\left(22.2_{-1.2}^{+1.1}\right.$ pc; Dupuy et al. 2020) is composed of a 0.1 " equalmagnitude M7.5 binary (VHS 1256-1257A and VHS 1256-1257B; Stone et al. 2016) and a lower mass L7 companion (component b; Rich et al. 2016) located 8" away from the central pair. It is one of the few systems in which all three components are substellar (Bouy et al. 2005; Radigan et al. 2013). The masses of the central pair components are estimated to be 50-90 $\mathrm{M}_{J u p}$ each and 10-35 
$\mathrm{M}_{\text {Jup }}$ for the L7 companion (Gauza et al. 2015; Rich et al. 2016; Stone et al. 2016; Guirado et al. 2018; Dupuy et al. 2020). This locates VHS 1256-1257b on the planet-brown dwarf boundary.

The strong lithium depletion observed in the high resolution spectra of the central pair and its kinematic membership to the Local Association implied an age of 150-300 Myr (Gauza et al. 2015; Rich et al. 2016; Stone et al. 2016). This young age, together with the 102 AU separation between the central pair and the L7 object, makes VHS 1256-1257 one of the most suitable system to search for debris disk around UCDs and exoplanets. Sub-mm observations could probe not only the emission of cold dust surrounding the central binary but also detect a dusty disk surrounding an L-type object, as suggested for others like G196-3B (Zakhozhay et al. 2017).

Previous radio observations of VHS 1256-1257 have shown emission coincident with the central binary at $8.4 \mathrm{GHz}$ (peak density of $\sim 60 \mu \mathrm{Jy} /$ beam; Guirado et al. 2018) while no detection at $1.4 \mathrm{GHz}$. The inferred spectral index of $\alpha=-1.1 \pm 0.3$ between $8 \mathrm{GHz}$ and $12 \mathrm{GHz}$ is indicative of non-thermal, optically thin, synchrotron, or gyrosynchrotron radiation. Were the $1.4 \mathrm{GHz}$ nondetection due to self-absorption, the magnetic field present in the M7.5 binary would be of 1.2-2.2 $\mathrm{kG}$ with a turnover frequency located between 5.0-8.5 GHz. No radio emission was found at the expected position of the $\mathrm{L} 7$ object, with a $3 \sigma$ upper limit of $9 \mu \mathrm{Jy}$ at $10 \mathrm{GHz}$.

Here we will present Very Large Array (VLA), European very-long-baseline interferometry (VLBI) Network (EVN), enhanced Multi Element Remotely Linked Interferometer Network (eMerlin), NOrthern Extended Millimeter Array (NOEMA), and Atacama Large Millimetre Array (ALMA) observations of the binary VHS 1256-1257AB. Additionally, we re-analysed VLA public data of this system (program 18A-430).

The great utility of radio observations is amplified in the case of binary systems as its submas astrometry can determine the dynamical masses which are crucial for benchmarks in low-mass evolutionary models (e.g. Azulay et al. 2017). These models are used to predict the masses of young stars and exoplanets from observational estimates of their luminosities and effective temperatures. However, there are some important discrepancies between observations and models for low-mass objects (e.g.: Pegues et al. 2021) which have been attributed (among other things) to the scarcity of well-known dynamical masses of such objects (Hillenbrand et al. 2004, Mathieu et al. 2007, Pegues et al. 2021). In this sense, we will also present here preliminary results of our VLA and EVN campaings targeted towards M dwarfs systems and UCDs, respectively. The former is an exploratory campaign of binary systems (see Table 1) with known age (as they belong to the $\mathrm{AB}$ Dor moving group) during 2 hours, while the latter is centered around four UCDs with previous VLA detections but never studied at VLBI resolution (see Table 2).

Table 1. Low-mass binary systems.

\begin{tabular}{|c|c|c|c|c|}
\hline Name & $d(p c)$ & Spectral Type & Sep & Ref \\
\hline \hline 2MASS J01225093-2439505 & 33.9 & $\mathrm{M} 3.5+\mathrm{L} 5$ & $1.45^{\prime \prime}$ & 1,2 \\
\hline 2MASS J07285137-3014490 & 15.7 & $\mathrm{M} 1+\mathrm{M} 3$ & $0.17^{\prime \prime}$ & $3,4,5$ \\
\hline 2MASS J22232904+3227334 & 15.1 & $\mathrm{M} 3+?$ & $1.3^{\prime \prime}$ & 3,9 \\
\hline 2MASS J22501512+2325342 & 57.3 & $\mathrm{M} 3+\mathrm{L} 3$ & $8.9^{\prime \prime}$ & 1,2 \\
\hline 2MASS J23513366+3127229 & 43.1 & $\mathrm{M} 2+\mathrm{L} 0$ & $2.4^{\prime \prime}$ & 1,8 \\
\hline
\end{tabular}

References: 1- GAIA DR2, 2- Bowler et al. (2013), 3- Koen et al. (2010), 4- Daemgen et al. (2007), 5- Rodet et al. (2018), 6-West et al. (2015), 7- Desrochers et al. (2018), 8- Bowler et al. (2012), 9- Mason et al. (2001) 
Table 2. UCDs observed with the EVN and their previous radio detections

\begin{tabular}{|c|c|c|c|}
\hline Name & Spectral Type & $F_{v}(\mu J y)^{1}$ & Ref \\
\hline \hline EQ J1122+2550 & T6 & $77 \pm 4$ & 1,2 \\
\hline LSR J1835+3259 & M8.5 & $525 \pm 15$ & 3 \\
\hline LSPM J0036+1821 & L3.5 & $259 \pm 19$ & 4,5 \\
\hline LP 349-25 & M8+M9 & $320 \pm 21$ & 6 \\
\hline
\end{tabular}

${ }^{1}$ VLA Density flux at $5 \mathrm{GHz}$ except for LSR J1835+3259 (at 8.5 GHz). References: 1- Williams et al. (2017), 2- Route and Wolszczan (2016), 3- Berger et al. (2006), 4- Forbrich \& Berger, 5- Berger et al. (2005), 6- Osten et al. (2007).

\section{Observations}

VHS 1256-1257 was observed during 2 years at a wide range of frequencies, which together with the data from Guirado et al. (2018) cover from $1.4 \mathrm{GHz}$ up to $345 \mathrm{GHz}$. Details of the observation log can be found in Table 3. Further details of the observations and data reduction are provided in Climent et al. (2022).

Table 3. Log of observations of the VHS 1256-1257 system

\begin{tabular}{cccccc}
\hline \hline Array & Frequency $(\mathrm{GHz})$ & Observing Date & UT range & Beam size & $\begin{array}{c}1 \sigma \mathrm{rms} \\
(\mu \mathrm{Jy} / \mathrm{beam})\end{array}$ \\
\hline eMerlin $^{\mathrm{a}}$ & 5.0 & 21 Oct. 2017 & $06: 17-17: 39$ & & \\
& & 22 Oct. 2017 & $06: 35-18: 57$ & $0.20^{\prime \prime} \times 0.05^{\prime \prime}$ & 20 \\
& 23 Oct. 2017 & $06: 51-18: 58$ & & \\
EVN $^{\mathrm{b}}$ & 5.0 & 23 Oct. 2018 & $06: 00-14: 00$ & $2.46 \times 7.82$ mas & 10 \\
VLA $^{\mathrm{c}}$ & 6.0 & 24 Oct. 2018 & $06: 00-14: 00$ & & \\
& & 13 Apr. 2018 & $02: 19-06: 19$ & $0.69^{\prime \prime} \times 0.35^{\prime \prime}$ & 3 \\
VLA & 33.0 & 17 Nov. 2018 & $17: 43-18: 43$ & $1.07^{\prime \prime} \times 0.69^{\prime \prime}$ & 7 \\
NOEMA & 230 & 26 Nov. 2018 & $14: 28-15: 28$ & & \\
ALMA & 345 & 7 Mar. 2019 & $05: 22-06: 25$ & $0.91^{\prime \prime} \times 0.81^{\prime \prime}$ & 40 \\
\hline
\end{tabular}

Notes. ${ }^{a}$ eMerlin antennas: Lovell, Mark II, Pickmere, Darnhall, Knockin, Defford, and Cambridge.

${ }^{\mathrm{b}}$ European VLBI Network using the following antennas: Jodrell Bank, Westerbork, Effelsberg, Medicina, Noto,

Onsala, Torun, Yebes, Hartebeesthoek, Cambridge, Darnhall, Defford, Kunming, and Pickmere.

${ }^{\mathrm{c}}$ Public data: program 18A-430.

VLA data of the low-mass binary systems of Table 1 were obtained during May 2019 with the VLA in B configuration at $8.4 \mathrm{GHz}$. The EVN observations of the objects presented in Table 2 were taken during March 2019 at $5 \mathrm{GHz}$. Detailed explanations of the observations and data reduction of these datasets will be published in the near future. 


\section{Results and discussion}

\subsection{VHS 1256-1257}

We analysed multi-band, multi-epoch data of VHS 1256-1257 (see Table 3) and found new detections of the central binary at $6 \mathrm{GHz}$ and $33 \mathrm{GHz}(73 \pm 4 \mu \mathrm{Jy}$ and $83 \pm 13 \mu \mathrm{Jy}$, respectively) with no detectable circular polarization, pulses, or variation during the observations. The emission at $33 \mathrm{GHz}$ appears stable over a period of at least 9 days whereas that at $6 \mathrm{GHz}$ is compatible with stability for almost 3 years.

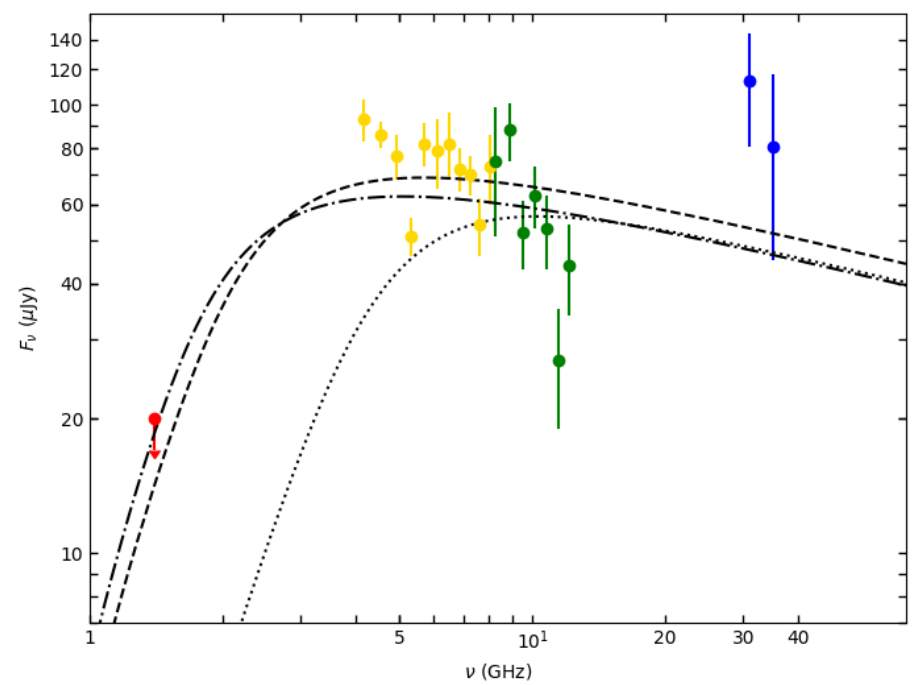

Figure 1: Total flux density of VHS 1256-1257AB from VLA observations (circles). Different colors indicate different bands: $1.4 \mathrm{GHz}$ (red), $6 \mathrm{GHz}$ (yellow), $10 \mathrm{GHz}$ (green), and $33 \mathrm{GHz}$ (blue). Lines represent different gyrosynchrotron models with fixed $\delta=1.36$ : dotted line for localized emitting region at the stellar surface with size of $1 \mathrm{R}_{*}$, dashed and dash-dot lines for radiation belts with maximum magnetic field strength at the surface level $\mathrm{B}_{0}=2000 \mathrm{G}$ and $\mathrm{B}_{0}=3000 \mathrm{G}$, respectively.

As can be appreciated in Fig. 1, the $33 \mathrm{GHz}$ detection is remarkable since the gyrosynchrotron model predicts non-detectable fluxes at such frequency. Complementing these detections, we found no detectable emission with VLBI arrays, ALMA or NOEMA. The lack of compact radio emission points to a scenario where both components emit with a flux ratio $<2.7$ (which in not unexpected as both have the same spectral type, mass and age) and so the contributions of each component of the binary may be very similar, whereas the non-detections with ALMA and NOEMA dismiss the possibility of a large debris disk (see Fig. 2). Combining all the constraints from different observations we considered a great numbers of scenarios: emission originating at one/both components, non-thermal gyrosynchrotron, thermal bremsstrahlung, optically thick thermal gyroresonance... We conluded that the most likely scenario that agrees with all the constraints is that of non-thermal gyrosynchrotron emission from a power-law energy distribution of electrons originating at radiation belts around both components. In order to explain the lack of rotational modulation, these radiation belts must be viewed equatorially. As a proof of feasibility, we simulated such scenario and found that the radiation belts would have a low plasma density (electron density $\mathrm{n}_{e}=300-700 \mathrm{~cm}^{-3}$ ), a moderate magnetic field strength $(\mathrm{B} \approx 140 \mathrm{G}$, assuming a $\sim \mathrm{kG}$ maximum strength on the stellar 


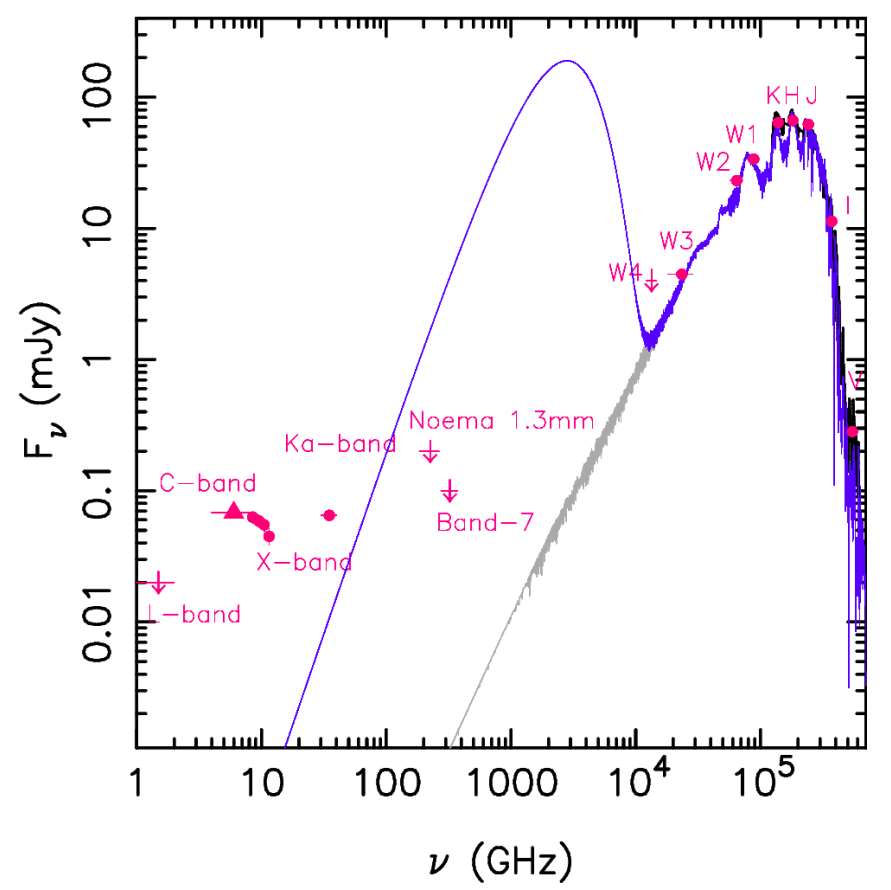

Figure 2: Spectral energy distribution of the unresolved binary VHS 1256-1257 AB from $1 \mathrm{GHz}$ up to optical wavelengths. Photometric observations are plotted as solid circles (detections) and arrows (2- $\sigma$ upper limits). The optical and near-infrared observed spectra (Gauza et al. 2015) are shown with a solid black line. The BT-Settl photospheric model (Baraffe et al. 2015) normalized to the J-band flux of VHS 1256-1257 AB is plotted as a gray line. This model corresponds to a cool dwarf with solar metallicity, $\mathrm{T}_{\text {eff }}$ $=2600 \mathrm{~K}$, and $\log \mathrm{g}=5.0\left[\mathrm{~cm} \mathrm{~s}^{-2}\right]$, which are the parameters expected for an M7.5 source with an age of a few hundred Myr (Chabrier et al. 2000). This photospheric flux density is combined with the emission of putative thin dusty disk with the parameters of the famous AU Mic debris disk (Liu et al. 2004), i.e., 0.89 $\mathrm{M}_{\text {lunar }}$, grain size of $100 \mu \mathrm{m}$, and temperature of $40 \mathrm{~K}$ (blue solid line).

surface), and with $\delta$ fixed at 1.36 (where $d N / d E \propto E^{-\delta}$ represents a power-law energy distribution of electrons). These numbers are not quantitative but show that such scenario is possible with reasonable parameter values.

These interesting results, however, can be affected by temporal variability as they are extracted in part from a non-simultaneous spectral energy distribution (SED) that covers almost 4 years. Additionally, while the proposed mechanism is the most likely, it is not the only possibility. Our analysis shows that a combination of optically thick gyroresonance and non-thermal gyrosynchrotron can also reproduce the SED while agreeing with the rest of constraints. As such, new quasisimultaneous observations will be crucial.

\subsection{Preliminary results on low mass binaries}

Out of the five binaries observed (Table 1), we successfully detected radio emission in three of them. A detailed presentation of the results and discussion will be published soon (Climent et al. in prep).

J0728-3014 is detected as an unresolved source with a Stokes I flux density of $180 \pm 2 \mu \mathrm{Jy}$ and no detectable circular polarization (see Fig. 3). A burst of radio emission is seen during the 

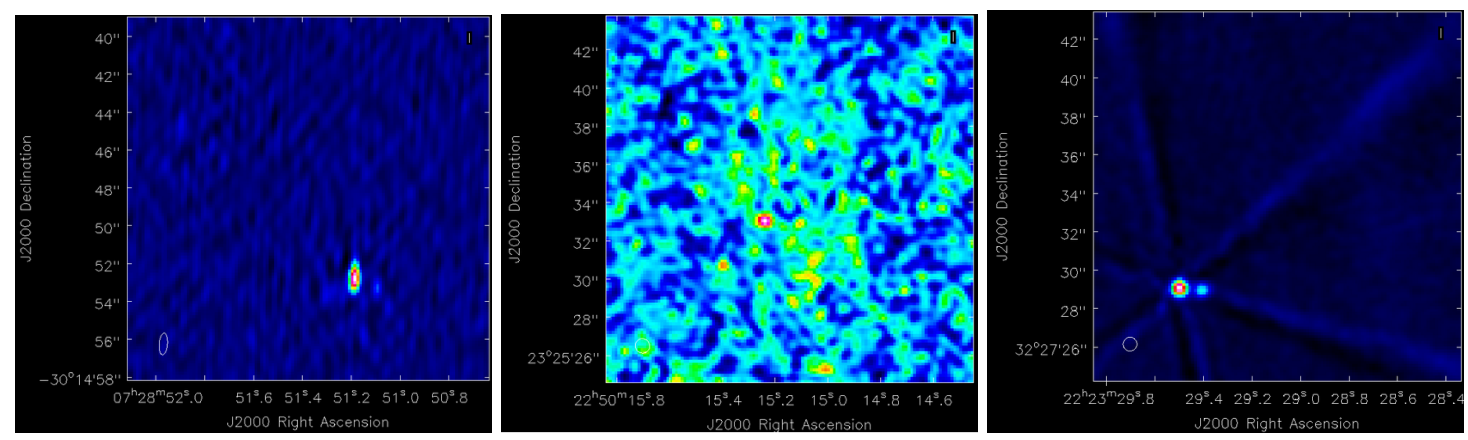

Figure 3: VLA detections of the low-mass binary objects of Table 1. From left to right: J0728-3014, $\mathrm{J} 2250+2325$, and $\mathrm{J} 2223+3227$.

last minutes 10 minutes of our observation which reaches $\sim 800 \mu \mathrm{Jy}$. Remarkably, this burst is not circularly polarized.

In spite of being the furthest object, we also found radio emission originating at the expected position of J2250+2325 with a Stokes RR flux density of $26 \pm 4 \mu \mathrm{Jy}$ (see Fig. 3). This unresolved source emits with $100 \%$ circular polarization despite no burst being identified during the observation.

Finally, J2223+3227 is resolved in our data (see Fig. 3) with Stokes I density fluxes of $445 \pm 4 \mu \mathrm{Jy}$ and $206 \pm 4 \mu \mathrm{Jy}$ for the primary and secondary components, respectively. During the second hour of the observation, the primary shows a burst of $\sim 1.4 \mathrm{mJy}$ although no significant circular polarization is detected.

\subsection{Preliminary results on compact radio emission of UCDs}

Preliminary images are shown in Fig. 4, with main results summarized in turn:

- All the members of our small sample (four objects, including two brown dwarfs, covering spectral types from M7 to T6) have been detected with the EVN, showing compact radio emission. Moderate to high level of circular polarization is present in at least two sources (EQ J1122+2550 and LSR J1835+3259).

- LP 349-25 is a binary UCD with known radio emission, but elusive (to our knowledge) to VLBI arrays. Our $2 \mathrm{hr}$-long EVN observations show the detection of only one component of LP 349-25. However, thanks to the sub-milliarcsecond positional precision provided by our EVN data, we could successfully analyze archival data of this system (GH009 project; $5 \mathrm{hr}$ duration); we notice that both components A and B are seen in the GH009 map (see Fig. 4), in excellent coincidence with the (relative) position predicted by the (relative) orbit of Dupuy et al. (2017).

- The detection of the very cool T6 brown dwarf EQ J1122+2550 is remarkable. The emission is highly polarized (RCP) with evident variability within the duration ( $2 \mathrm{hr}$ ) of our observations; in principle, this would be compatible with (auroral) electron cyclotron maser (ECM) emission (see Williams et al. 2017). EQ J1122+2550 is one of the few T-objects detected in radio waves, and, to our knowledge, the first one with VLBI arrays. 

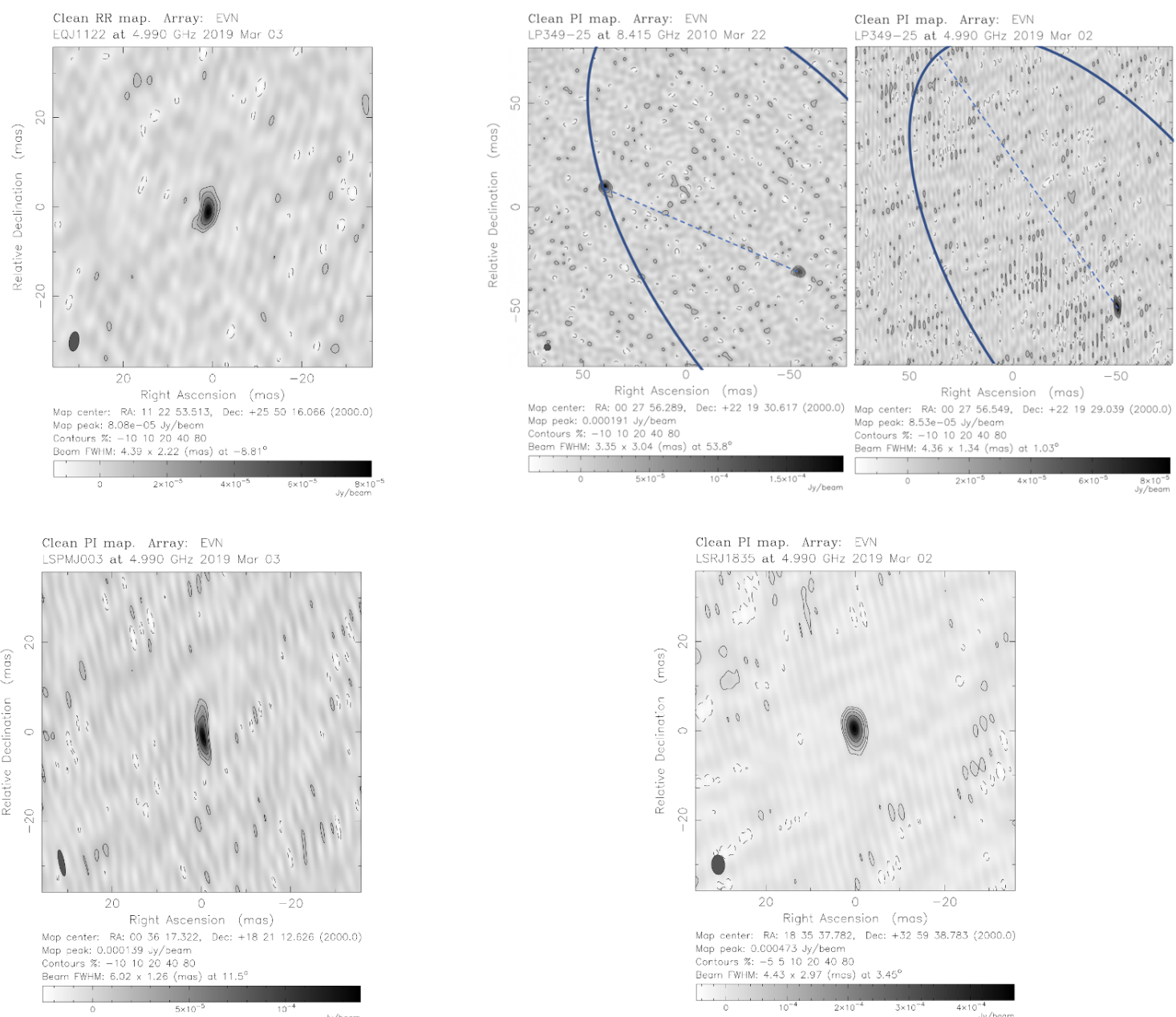

Figure 4: EVN images of the UCDs observed in EC067 (see Table 2): EQ J1122+2550 (top left), LP 349-25 (top right; two maps, see below), LSPM J0036+1821 (bottom left), and LSR J1835+3259 (bottom right). For LP 349-25, the resulting map of archival 8.4 GHz data (GH009; epoch 2010.22; both components detected) is shown along with the EC067 map (only A component visible); the relative orbit from Dupuy et al. (2017) is shown in continuos line; dashed lines connect the predicted relative position of both components.

New, more detailed observations have been taken of these four UCDs. The results will be published in the near future (Climent et al. in prep).

\section{Conclusions}

Radio observations are a great tool to better understand to exciting filed of UCDs and also to provide robust, much-needed dynamical masses of low-mass systems in order to calibrate evolutionary models. We have shown that a detailed study of the radio emission present in one of these objects, VHS 1256-1257, has allowed us to dismiss various emission mechanisms pointing to non-thermal gyrosynchrotron emission originating at radiation belts as the most likely one.

We also presented preliminary results from our observations with the VLA and EVN. The first ones have successfully detected radio emission from 3 binary systems which we will observe at 
higher angular resolution with VLBI in order to estimate their dynamical masses. The second ones have more than doubled the number of UCD with published detection at VLBI resolution and show an exciting field of study that we will explore in the near future.

\section{References}

[1] Azulay, R., Guirado, J. C., Marcaide, J. M., et al. 2017, A\&A, 607, A10

[2] Baraffe, I., Homeier, D., Allard, F., \& Chabrier, G. 2015, A\&A, 577, A42

[3] Berger et al. 2001, Natur , 410, 338

[4] Berger, E. 2002, ApJ, 572, 50

[5] Bouy, H., Martín, E. L., Brandner, W., \& Bouvier, J. 2005, AJ, 129, 511

[6] Chabrier, G., Baraffe, I., Allard, F., \& Hauschildt, P. 2000, ApJ, 542, 464

[7] Climent, J. B., Guirado, J. C., et al. 2022, arXiv:2201.12606 (accepted in A\&A).

[8] Curiel, S., Ortiz-León, G. N., Mioduszewski, A. J., \& Torres, R. M. 2020, AJ, 160, 97

[9] Dupuy, T. J. \& Liu, M. C. 2017, ApJS, 231, 15

[10] Dupuy, T. J., Liu, M. C., Magnier, E. A. et al 2020, RNAAS, 4, 54

[11] Forbrich J., Berger E., 2009, ApJ, 706, L205

[12] Forbrich, J., Dupuy, T. J., Reid, M. J., et al. 2016, ApJ, 827, 22

[13] Gauza, B., Béjar, V. J. S., Pérez-Garrido, A., et al. 2015, ApJ, 804, 96

[14] Guirado et al. 2018, A\&A, 610, A23

[15] Hallinan, G., Bourke, S., Lane, C., et al. 2007, ApJ, 663, L25

[16] Hallinan et al. 2008, ApJ, 684, 644

[17] Hillenbrand, L. A., \& White, R. J. 2004, The Astrophysical Journal, 604, 741

[18] Kao et al. 2016, ApJ, 818, 24

[19] Kao et al. 2018, ApJS, 237, 25

[20] Liu, M. C. 2004, Science, 305, 1442

[21] Mathieu, R. D., Baraffe, I., Simon, M., Stassun, K. G., \& White, R. 2007, in Protostars and Planets V, ed. B. Reipurth, D. Jewitt, \& K. Keil, 411

[22] Pegues, J., Czekala, I., Andrews, S. M., et al. 2021, ApJ, 908, 42

[23] Rich, E. A., Currie, T., Wisniewski, J. P., et al. 2016, ApJ, 830, 114 
[24] Radigan, J., Jayawardhana, R., Lafreniére, D., et al. 2013, ApJ, 778, 36

[25] Reiners, A., \& Basri, G. 2010, ApJ, 710, 924

[26] Stone, J. M., Skemer, A. J., Kratter, K. M., et al. 2016, ApJ, 818, L12

[27] Turnpenney, S., Nichols, J. D., Wynn, G. A., \& Burleigh, M. R. 2018, ApJ, 854, 72

[28] Turnpenney, S., Nichols, J. D., Wynn, G. A., et al. 2020, MNRAS, 494, 5044

[29] Vedantham, H. K., Callingham, J. R., Shimwell, T. W., et al. 2020, ApJ, 903, L33

[30] Zakhozhay, O. V., Zapatero Osorio, M. R., Béjar, V. J. S., \& Boehler, Y. 2017, MNRAS, 464, 1108

[31] Zhang, Q., Hallinan, G. et al. 2020, ApJ, 891, 1 